\title{
The prediction of myometrial infiltration by three-dimensional ultrasonography in patients with endometrial carcinoma: a validation study from Ege University Hospital.
}

\author{
Mete Ergenoglu$^{1}$, Levent Akman ${ }^{1}$, Mustafa Cosan Terek ${ }^{1}$, Cem Yasar Sanhal ${ }^{1}$, Ozgur Yeniel' \\ Ozgul Vupa Cilengiroglu², Ahmet Aydin Ozsaran'1, Yilmaz Dikmen', Osman Zekioglu³
}

${ }^{1}$ Ege University Medical School, Department of Obstetrics and Gynecology, ${ }^{2}$ Dokuz Eylul University, Faculty of Sciences, Department of Statistics, ${ }^{3}$ Ege University Medical School, Department of Pathology, Izmir, Turkey

\begin{abstract}
Aim: To predict the myometrial invasion with three-dimensional (3D) ultrasonography in a cohort of patients with endometrial carcinoma by a previously described technique. Material and methods: The moyometrial infiltration was evaluated by $3 \mathrm{D}$ ultrasonography before surgery in 54 patients with endometrial carcinoma. After scanning the whole uterus by ultrasonography, three perpendicular planes were identified to find the shortest myometrial tumor-free distance to serosa (TDS) by examining the lateral, anterior, posterior, and fundal parts of the myometrium. Myometrial infiltration was also estimated by the subjective impression of the examiner. The reference standards consist of myometrial infiltration and TDS which are measured by an experienced pathologist. Results: Forty-five patients (age range 45-86 years) were included for the final analysis. Myometrial invasion was $<50 \%$ in 36 and $\geq 50 \%$ in 9 cases at histologic sections. The TDS which is measured with $3 \mathrm{D}$ ultrasonography was positively correlated with histologically measured TDS $(\mathrm{r}=0.474, \mathrm{p}=0.001)$. The best cut-off value for ultrasonographically measured TDS was $9 \mathrm{~mm}$ with a sensitivity of $89 \%$, specifity of $61 \%$, positive predictive value of $36 \%$, and negative predictive value of $96 \%$. Subjective impression has a sensitivity of $100 \%$, specifity of $88 \%$, positive predictive value of $69 \%$, and negative predictive value of $100 \%$. Cervical involvement was correctly identified in all 6 cases by subjective impression. Conclusion: This validation study confirms the 3D ultrasonography as a valuable tool for the evaluation of myometrial infiltration in patients with endometrial carcinoma.
\end{abstract}

Keywords: endometrial carcinoma, myometrial infiltration, 3D ultrasonography

\section{Introduction}

The surgical staging of endometrial carcinoma patients is an essential part of the overall therapy [1]. Pelvic and para-aortic lymphadenectomy is an important part of the complete surgical staging with an inherent complication rate. Retroperitoneal lymphadenectomy may be avoided in low-risk patients. Myometrial infiltration of the tumor is one of the factors that define low-risk pa-

Received 07.11.2015 Accepted 31.01.2016

Med Ultrason

2016, Vol. 18, No 2, 201-206

Corresponding author: Levent Akman, MD

Ege University Medical School

Department of Obstetrics and Gynecology

35100, Bornova, Izmir, Turkey

Phone: +90-232-3901730

E-mail:leventakman@gmail.com tients along with tumor type and histologic grade [2]. Myometrial infiltration of the tumor can be predicted by magnetic resonance imaging (MRI) and ultrasonography (US) in the preoperative setting. Macroscopic evaluation of the uterus and frozen section analyses are done intraoperatively to predict the myometrial invasion [3]. The accuracy of MRI imaging for the assessment of myometrial invasion in patients with endometrial carcinoma is reported between $62 \%$ to $95 \%$ in two studies $[4,5]$.

Transvaginal US is a method to examine the myometrial invasion and the cervical stromal invasion non-invasively, without using contrast agent, in patients with endometrial carcinoma. Three-dimensional (3D) US adds a more accurate examination of the uterus with many perpendicular planes [6]. Raine-Fenning et al [7] and Alcazar et al [8] reported 3D US as less operator dependent than two-dimensional US. 
Alcazar et al $[8,9]$ proposed a technique for assessing the myometrial invasion in patients with endometrial carcinoma. The aim of the present study was to validate the proposed technique in a cohort of patients with endometrial carcinoma.

\section{Materials and methods}

\section{Study Patients}

The Ege University Ethical Committee approval was obtained and written consent was obtained from all patients for the study. Patients with presumed early stage endometrial carcinoma were included in the study, in a single University Hospital. Preoperative work-up of the patients consists of chest X-ray, whole abdominal ultrasonography, blood biochemical tests, physical and gynecological examination. The diagnosis of endometrial carcinoma was obtained either by endometrial curettage or hysterescopic endometrial biopsy. The patients with intramural leiomyoma greater than $3 \mathrm{~cm}$ and submucous leiomyoma $(n=6)$ were excluded from the study. Surgical treatment and staging was not performed because of cardiac disease $(n=1)$, parametrial involvement with intraabdominal carcinomatosis $(\mathrm{n}=1)$ and parametrial involvement $(\mathrm{n}=1)$ in three patients.

\section{Ultrasonography}

All ultrasonographic examinations were performed within two weeks prior surgery with a transvaginal probe (3-9 MHz, E8, GE Healthcare, Milwaukee, WI). All ultrasonographic examinations were performed by one physician (A.M.E.) with three years of experience in 3D US. Two-dimensional US examination was initially performed to explore the uterus, adnexa and endometrial thickness. The 3D volume box was placed over the endometrial and myometrial areas. A total of 4 volumes were obtained and stored on a hard disc for further evaluation [8]. Volume examinations were blinded to histological results. Myometrial infiltration was assessed in the stored volume. Transverse, sagittal, and coronal planes of the uterus were evaluated carefully. Myometrial tumor-free distance to serosa was measured in the lateral walls and fundus in coronal and sagittal planes (fig 1). The subjective shortest tumor-free distance to serosa (TDS) which was found in any area was selected to determine the depth of myometrial infiltration. Myometrial infiltration was also estimated subjectively according to the examiner impression of the depth of invasion $(\geq 50 \%$ or $<50$ $\%$ ) by identifing the point in which the myometrial-endometrial interface was not clearly defined. The examiner compared the supposedly tumor-free myometrial wall at that point with the opposite myometrial wall: if a marked asymmetry was identified, deep ( $\geq 50 \%$ ) infiltration was

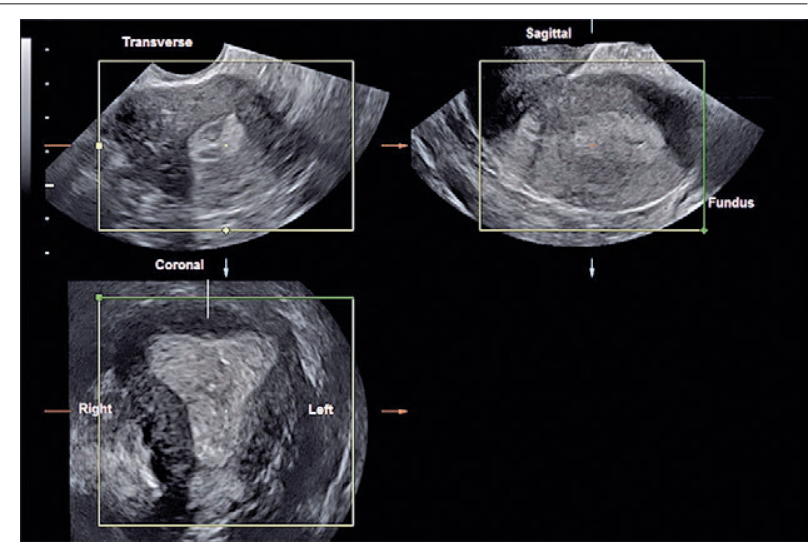

Fig 1. Three-dimensional ultrasonography images in a case of endometrial cancer in transverse, sagittal and coronal planes.

documented; if myometrial thickness was similar in both myometrial walls, superficial $(<50 \%)$ infiltration was documented. The involvement of the lower uterine segment of uterus and cervical canal was evaluated subjectively by $3 \mathrm{D}$ US. The subjective disruption of the lower uterine segment of uterus and cervical canal with irregular appearance of endometrial-myometrial border was determined as subjective cervical involvement of endometrial carcinoma.

\section{Control patients}

Two control groups of asymptomatic patients were recruited to determine the normal TDS value in normal uteri. The first group included premenopausal patients $(n=20)$ and the second group postmenopausal women $(n=20)$. The postmenopausal period was defined as an absence of menstruation for 1 year in women older than 45 years of age. None of these two groups of patients had uterine or endometrial pathologies including leiomyomas, adenomyosis, congenital anomalies, endometrial polyps, endometrial hyperplasia and endometrial thickness $>5 \mathrm{~mm}$ in postmenopausal age. None of these patients used oral contraceptives, hormone replacement therapy, or tamoxifen. Reproducibility studies of TDS measurement were performed previously by Alcazar et al [9].

\section{Surgical staging}

Standard surgery included total abdominal hysterectomy, bilateral salpingo-oophorectomy, and pelvic washings for cytological examination. Omentectomy was performed in serous papillary and clear cell histological types of endometrial carcinoma. Pelvic and paraaortic lymphadenectomy was performed in grade 3 , serous papillary and clear cell histological types and outer myometrial infiltration $(\geq 50 \%)$ on frozen section pathological analysis. The pathological data from an individual patient included histological type, histological grade, depth of myometrial infiltration, lymphovascular space in- 
Med Ultrason 2016; 18(2): 201-206

volvement, TDS, cervical involvement, and lymph node status. The pathological data were used as the reference standard of the study. All surgeries were performed by experienced gynecologic oncologists with dedicated surgical and pathological teams in a specialized gynecologic oncology division of University Hospital. Tumor stage was determined according to the International Federation of Gynecology and Obstetrics Criteria [1]. Tumor histologic grade was determined according to the following three-grade system: grade 1 carcinoma showed solid growth pattern in less than $5 \%$ of the tumor; grade 2 carcinoma showed solid growth pattern in 5\%-50\% of the tumor; grade 3 carcinoma showed solid growth pattern in more than $50 \%$ of the tumor.

\section{Statistical Analysis}

The Kolmogorov-Smirnov test was used to assess the normal distribution of continuous data. Continuous data were compared by using one-way analysis of variance when data were normally distributed or by using the MannWhitney $U$ test when data were not normally distributed. The $t$ test was used to compare the means for two independent samples. These tests were used for comparing TDS measured by using ultrasonography in the control group according to the site of measurement, and TDS measured by using ultrasonography in study group according to histologic depth of invasion. A receiver operating characteristic (ROC) curve was plotted to determine the best cutoff value for TDS for identifying deep myometrial invasion. The best cutoff value was chosen according to the best sensitivity with the lowest false-positive rate. A p value of 0.05 or less was considered to indicate a statistically significant difference. All analyses were performed by using software (SPSS, version 15.0; SPSS, Chicago, Ill).

\section{Results}

The mean age of the patients with endometrial carcinoma was $62.1 \pm 10(45-86)$ and $89 \%(n=40)$ of women were
Table I. Histologic features of endometrial cancer.

\begin{tabular}{ll}
\hline Clinical Features & Patients (n, \%) \\
\hline Histologic type & $38(84.6 \%)$ \\
Endometrioid & $4(8.8 \%)$ \\
Serous papillary & $3(6.6 \%)$ \\
Clear cell & $18(40 \%)$ \\
Histologic grade & $21(46.7 \%)$ \\
G1 & $6(13.3 \%)$ \\
G2 & $36(80 \%)$ \\
G3 & $9(20 \%)$ \\
Myometrial infiltration & \\
$<50 \%$ & $33(73.4 \%)$ \\
$\geq 50 \%$ & $6(13.3 \%)$ \\
Tumor stage & $6(13.3 \%)$ \\
IA & \\
IB &
\end{tabular}

postmenopausal. Tumor histologic features are shown in Table I. Table II shows median TDS at the level of fundus, anterior and posterior uterine walls, and lateral uterine wall in the control groups. TDS in all uterine walls was longer in premenopausal women than in postmenopausal women.

The Mann-Whitney U test was used to compare TDS measured by 3D ultrasonography and by a pathologist according to myometrial infiltration. Median TDS measured by a pathologist and that measured by using threedimensional ultrasonography were significantly shorter in those tumors with $50 \%$ or more myometrial infiltration than in those with less than $50 \%$ infiltration (Table III).

There was a positive correlation between pathologically measured and 3D ultrasonographically-measured TDS $(r=0.474, p=0.001)$; mean and standard deviations of these measurements were found as 9.5, 3.27 and 14.5, 8.27 (mean, standard deviation), respectively. There was a statistically significant difference between $3 \mathrm{D}$ ultrasonographically-measured TDS and histologically measured TDS ( $\mathrm{p}=0.001)$. Median TDS in the control group

Table II. Measurement of median tumor-free distance to serosa (TDS) at the level of fundus, anterior and posterior uterine walls, and lateral uterine wall in control groups.

\begin{tabular}{|c|c|c|c|c|}
\hline & Fundus (mm) & Lateral uterine wall $(\mathrm{mm})$ & Anterior uterine wall (mm) & Posterior uterine wall (mm) \\
\hline Premenopausal $(n=20)$ & $18(14-24)$ & $17(11-27)$ & $18(12-29)$ & $19(11-30)$ \\
\hline Postmenopausal $(\mathrm{n}=20)$ & $10(5-13)$ & $11(8-14)$ & $11(8-13)$ & $12(9-16)$ \\
\hline$P$ value & 0.001 & 0.002 & 0.002 & 0.009 \\
\hline
\end{tabular}

Data are medians, with ranges in parentheses, $n-$ number of patients

Table III. Tumor-free distance measured by 3D ultrasongraphy and histology.

\begin{tabular}{llll}
\hline Parameter & 3D US-measured TDS (mm) & Histologically measured TDS (mm) & P value \\
\hline$<50 \%$ myometrial infiltration & $10(4.8-19.0)$ & $15(7.0-40.0)$ & 0.004 \\
$\geq 50 \%$ myometrial infiltration & $7(4.0-10.0)$ & $5(2.0-19.0)$ & 0.000 \\
\hline
\end{tabular}

Data are medians, with ranges in parentheses, TDS: tumor-free distance to serosa 


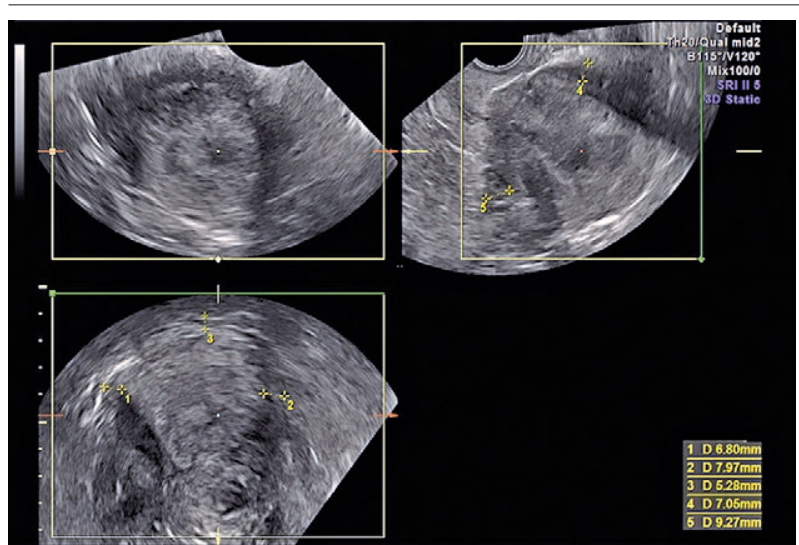

Fig 2. Three-dimensional ultarsonography image shows measurement of tumor-free distance to serosa (TDS) in case with deep myometrial infiltration. The shortest TDS was $5.28 \mathrm{~mm}$. (D: diameter)

Table IV. Diagnostic performance of tumor-free distance to serosa (TDS) as measured by 3D ultrasonography and the subjective impression of the examiner by ultrasonography.

\begin{tabular}{llll}
\hline Parameter & \multicolumn{3}{l}{ Myometrial infiltration } \\
& $\mathbf{2 5 0} \%$ & $<\mathbf{5 0} \%$ & Total \\
\hline TDS & & & \\
$\leq 9.0 \mathrm{~mm}$ & 8 & 14 & 22 \\
$>9.0 \mathrm{~mm}$ & 1 & 22 & 23 \\
Subjective impression & & & \\
$\geq 50 \%$ & 9 & 4 & 13 \\
$<50 \%$ & 0 & 32 & 32 \\
Total & 9 & 36 & 45 \\
\hline
\end{tabular}

TDS: Tumor-free distance

[14 mm (5-30)] was significantly higher than in the study group for tumors infiltrating less than $50 \%$ and for those infiltrating more than $50 \%$.

The receiver operating characteristic (ROC) curve determined that a shorter 3D ultrasonographically-measured TDS was a predictor for deep myometrial infiltration. The best cut-off value for 3D ultrasonographically-measured TDS was $9 \mathrm{~mm}$, which allowed for identification of cases with deep infiltration with $11 \%$ false negative and $39 \%$ false positive rate. Figure 2 shows a case of deep myometrial infiltration as determined by using $3 \mathrm{~d}$ US.

Only one case in the postmenopausal control group had a TDS less than $9 \mathrm{~mm}$ ( $5 \mathrm{~mm})$.

(Tables IV). For TD, sensitivity was $89 \%$, specificity $61 \%$, false positive rate $39 \%$, false negative rate $11 \%$. positive predictive value $36 \%$, and negative predictive value was $96 \%$. For subjective impression, sensitivity was $100.0 \%$. specificity $88.9 \%$, positive predictive value $69.0 \%$ and negative predictive value $100.0 \%$. With subjective impression, the false positive rate was $11.2 \%$, which was much lower than that of TDS $(p<0.001)$.
Cervical involvement was correctly identified in all of six $(100 \%)$ cases by subjective sonographic impression.

\section{Discussions}

Myometrial infiltration is one of the most important factors determining lymph node metastasis and adjuvant radiotherapy in patients with endometrial carcinoma. The reliable estimation of myometrial infiltration before surgery by a non-invasive methods is surely very helpful for the planning of surgical procedure and informing the patient about the extent of surgical procedure [10]. The lymphadenectomy procedure also has an inherent morbidity including lymphocele formation, vascular injury, and thrombosis [11]. Intraoperative gross evaluation of the uterus has a sensitivity of $67 \%-77 \%$ with a false negative rate for detection of deep myometrial invasion of about 23\%-33\% [3,12-14]. MRI has a sensitivity of $80 \%$ to $91 \%$ for preoperative evaluation of myometrial infiltration by endometrium carcinoma [4,15-17]. Also, adding diffusion weighted imaging sequence to the standard MRI, increases the detection of deep myometrial invasion of endometrial cancer [18]. However, MRI has a high cost and is not available in all institutions.

3D US provides multiplanar imaging of uterus in real-time including coronal planes. Alcazar et al [8] demonstrated that this technique is much less operator dependent than two-dimensional US. The sagittal plane of uterus can be assessed by two-dimensional US but it is almost impossible to gain knowledge regarding the coronal plane of the uterus which is easily visualized by $3 \mathrm{D}$ US.

Alcazar et al [9] were able to predict the deep myometrial infiltration in all cases of endometrial cancer by uterine virtual navigation. Their data indicated that with a $9.0 \mathrm{~mm}$ cut-off, TDS can accurately identify all cases of deep infiltration without any false-negative patients. They also showed that with subjective impression, the false-positive rate is $17.4 \%$. In the present study, with subjective impression, the false positive rate was $11.2 \%$ which is much lower than that of TDS $(p<0.001)$.

In line with the original study, we validated that the best cut-off value for 3D ultrasonographically-measured TDS was $9 \mathrm{~mm}$ for identifying myometrial infiltration of $50 \%$ or more [9]. Moreover in our study subjective impression had a better diagnostic performance than 3D ultrasonography in determining the deep myometrial infiltration.

However, false positive cases have less clinical importance from the perspective of oncological surgery. It is more important to leave the positive lymph nodes un- 
attended in cases with deep myometrial invasion. In line with the study of Alcazer et al [9] the low false negative rate rather than false positive rate and higher sensitivity of both subjective impression and TDS, 3D US is a good alternative to MRI.

TDS is important for predicting recurrence in endometrial cancer. Lindauer et al [19] determined whether a tumor-free distance from the uterine serosa is more predictive of patient outcome than the depth of endometrial cancer invasion into the myometrium. In a multivariate model, tumor-free distance from the uterine serosa was shown to correlate with surgicopathologic variables, recurrence risk, and survival.

Karlson et al [20] first suggested the use of the tumor/ uterine anteroposterior diameter ratio, a result of $>0.5$ indicating high risk of deep myometrial invasion, with a sensitivity of $79 \%$ and a specifity of $100 \%$. Mascilini et al [21] compared the diagnostic accuracy of subjective US assessment with that of objective measurement techniques in women with endometrial cancer. They found that the sensitivity (72\%) and specifity (76\%) of tumor/uterine anteroposterior diameter is not significantly different from those of subjective evaluation (sensitivity $77 \%$, specifity $81 \%$ ). They proposed the distance from outer cervical os to lower margin of tumor for predicting cervical invasion; however, it had only non-significantly higher sensitivity than subjective evaluation ( $73 \%$ vs $54 \%$ ).

Alcazar et al compared the diagnostic performance of six different approaches (impression of examiner, Karlsson's criteria, endometrial thickness, tumor/uterine 3D volume ratio, TDS and von Holsbeke's subjective model) for assessing myometrial infiltration using transvaginal/ transrectal US in endometrial carcinoma [22,23]. The study showed that the subjective impression seems to be the best approach for assessing myometrial infiltration in grade 1 or 2 endometrioid carcinoma and the use of mathematical models and other objective $2 \mathrm{D}$ and $3 \mathrm{D}$ measurement techniques do not improve diagnostic performance. Also, their metaanalysis showed that objective measurement techniques were not superior to subjective assessment [24].

The results on the use of US to assess cervical invasion are generally very good for subjective assessment, with sensitivities ranging from 77 to $93 \%$ and specifities ranging from 85 to $99 \%$ [25-27]. Subjective evaluation takes more features into account in addition to size and proportion, including regularity of the endometrial-myometrial border and vascular pattern. In large exophytic tumors, the tumor proportion will be great but the endometrial-myometrial border will be regular and the focal vessel pattern will indicate that it is a large polypoid lesion bulging into the cavity and stretching the surround- ing myometrium, rather than infiltrating [28]. In the present study, cervical involvement was correctly identified in all cases by subjective sonographic impression.

The limitation of the study was relatively few patient numbers. Further prospective studies with a larger population are required in order to determine a more accurate cut-off value for identifying myometrial infiltration of $50 \%$ or more in endometrial cancer.

In conclusion, tumors may infiltrate any region of the myometrium and 3D US allows the assessment of the uterus in all planes. In this present study, a subjective impression with 3D US determined deep myometrial infiltration in all cases. In line with the original study the best cut-off value for 3D ultrasonographically-measured TDS is $9 \mathrm{~mm}$ for identifying myometrial infiltration of $50 \%$ or more. However, more studies are required to prove the practicability of 3D US to the assessment of the depth of myometrial infiltration by endometrial cancer.

\section{Conflict of interest: none}

\section{References}

1. Creasman W. Revised FIGO staging for carcinoma of the endometrium. Int J Gynaecol Obstet 2009; 105: 109.

2. Aalders JG, Thomas G. Endometrial cancer: revisiting the importance of pelvic and paraaortic lymph nodes. Gynecol Oncol 2007; 104: 222-231.

3. Traen K, Hølund B, Mogensen O. Accuracy of preoperative tumor grade and intraoperative gross examination of myometrial invasion in patients with endometrial cancer. Acta Obstet Gynecol Scand 2007; 86: 739-741.

4. Rockall AG, Menoni R, Sohaib SA, et al. Evaluation of endometrial carcinoma on magnetic resonance imaging. Int $\mathrm{J}$ Gynecol Cancer 2007; 17: 188-196.

5. Torricelli P, Ferraress S, Fiocchi F, et al. 3-T MRI in the preoperative evaluation of depth of myometrial infiltartion in endometrial cancer. AJR Am J Roentgenol 2008; 190: 489-495.

6. Benacerraf BR, Shipp TD, Bromley B. Impoving the efficiency of gynecologic sonography with 3-dimensional volumes: a pilot study. J Ultrasound Med 2006; 25: 165-171.

7. Raine-Fenning NJ, Campbell BK, Clewes JS, Kendall NR, Johnson IR. The reliability of virtual organ computer- aided analysis (VOCAL) for the semiquantification of ovarian, endometrial and subendometrial perfusion. Ultrasound $\mathrm{Ob}-$ stet Gynecol 2003; 22: 633-639.

8. Alcázar JL, Merce LT, Manero MG, Bau S, Lopez-Garcia G. Endometrial volume and vascularity measurements by transvaginal 3-dimensional ultrasonography and power Doppler angiography in stimulated and tumoral endometria: an interobserver reproducibility study. J Ultrasound Med 2005; 24: 1091-1098.

9. Alcázar JL, Galvan R, Albela S, et al. Assessing myometrial infiltration by endometrial cancer: Uterine virtual navi- 
gation with three dimensional US. Radiology 2009; 250: 776-783.

10. Creasman WT, Morrow CP, Bundy BN, Homesley HD, Graham JE, Heller PB. Surgical pathologic spread patterns of endometrial cancer: a Gynecologic Oncology Group study. Cancer 1987; 60: 2035-2040.

11. Christensen JW, Dueholm M, Hansen ES, Marinovskij E, Lundorf E, Ortoft G. Assessment of myometrial invasion in endometrial cancer using three-dimensionalultrasound and magnetic resonance imaging. Acta Obstet Gynecol Scand 2016; 95: 55-64

12. Fischerova D, Frühauf F, Zikan M, et al. Factors affecting sonographic preoperative local staging of endometrial cancer. Ultrasound Obstet Gynecol 2014; 43: 575-585.

13. Eriksson LS, Lindqvist PG, Flöter Rådestad A, et al. Transvaginal ultrasound assessment of myometrial and cervical stromal invasion in women with endometrial cancer: interobserver reproducibility among ultrasound experts and gynecologists. Ultrasound Obstet Gynecol 2015; 45: 476-482.

14. Berretta R, Merisio C, Piantelli G, et al. Preoperative transvaginal ultrasonography and intraoperative gross examination for assessing myometrial invasion by endometrial cancer. J Ultrasound Med 2008; 27: 349-355.

15. Antonsen SL, Jensen LN, Loft A, et al. MRI, PET/CT and ultrasound in the preoperative staging of endometrial cancer - a multicenter prospective comparative study. Gynecol Oncol 2013; 128: 300-308.

16. Park JY, Kim EN, Kim DY, et al. Comparison of the validity of magnetic resonance imaging and positron emission tomography/ computed tomography in the preoperative evaluation of patients with uterine corpus cancer. Gynecol Oncol 2008; 108: 486-492.

17. Andreano A, Rechichi G, Rebora P, Sironi S, Valsecchi MG, Galimberti S. MR diffusion imaging for preoperative staging of myometrial invasion in patients with endometrial cancer: a systematic review and meta-analysis. Eur Radiol 2014; 24: 1327-1338.

18. Das SK, Niu XK, Wang JL, et al. Usefulness of DWI in preoperative assessment of deep myometrial invasion in patients with endometrial carcinoma: a systematic review and meta-analysis. Cancer Imaging 2014; 14: 32.

19. Lindauer J, Fowler JM, Manolitsas TP, et al. Is there a prognostic difference between depth of myometrial inva- sion and the tumor-free distance form the uterine serosa in endometrial cancer? Gynecol Oncol 2003; 91: 547-551.

20. Karlsson B, Norstrom A, Granberg S, Wikland M. The use of endovaginal ultrasound to diagnose invasion of endoemtrial carcinoma Ultrasound Obstet Gynecol 1992; 2: 35-39.

21. Mascilini F, Testa AC, Van Holsbeke C, Ameye L, Timmerman D, Epstein E. Evaluating myometrial and cervical invasion in women with endometrial cancer: comparing subjective assessment with objective measurement techniques Ultrasound Obstet Gynecol 2013; 42: 353-358.

22. Van Holsbeke C, Ameye L, Testa AC, et al. Development and external validation of new ultrasound-based mathematical models for preoperative prediction of high-risk endometrial cancer. Ultrasound Obstet Gynecol 2014; 43: 586-595.

23. Alcazar JL, Pineda L, Martinez-Astorquiza Corral T, et al. Transvaginal/transrectal ultrasound for assessing myometrial invasion in endometrial cancer: a comparison of six different approaches. J Gynecol Oncol 2015; 26: 201-207.

24. Alcázar JL, Orozco R, Martinez-Astorquiza Corral T, et al. Transvaginal ultrasound for preoperative assessment of myometrial invasion in patients with endometrial cancer: a systematic review and meta-analysis. Ultrasound Obstet Gynecol 2015; 46: 405-413.

25. Akbayır O, Corbacioglu A, Numanoglu C, et al. Preoperative assessment of myometral and cervical invasion in endometrial carcinoma by transvaginal ultrasound Gynecol Oncol 2011; 122: 600-603.

26. Savelli L, Ceccarini M, Ludovisi M, et al. Preoperative local staging of endoemtrial cancer: transvaginal sonography vs. magnetic resonance imaging. Ultrasound Obstet Gynecol 2008; 31: 560-566.

27. Sawicki W, Spiewankiewicz B, Stelmachow J, Cendrowski $\mathrm{K}$. The value of ultrasonography in preoperative assessment of selected prognostic factors in endometrial cancer. Eur J Gynaecol Oncol 2003; 24: 293-298.

28. Leone FP, Timmerman D, Bourne T, et al. Terms, definitions and measurements to describe the sonographic features of the endometrium and intrauterine lesions: a consensus opinion from the International Endometrial Tumor Analysis (IETA) group. Ultrasound Obstet Gynecol 2010; 35: 103-112. 